\title{
ARTÍCULO ORIGINAL: Servicios brindados a los usuarios mayores de 18 años con enfermedad oncológica terminal en el Centro Nacional del Control del Dolor y Cuidados Paliativos de Costa Rica, desde su ingreso hasta su fallecimiento, 2010-2012
}

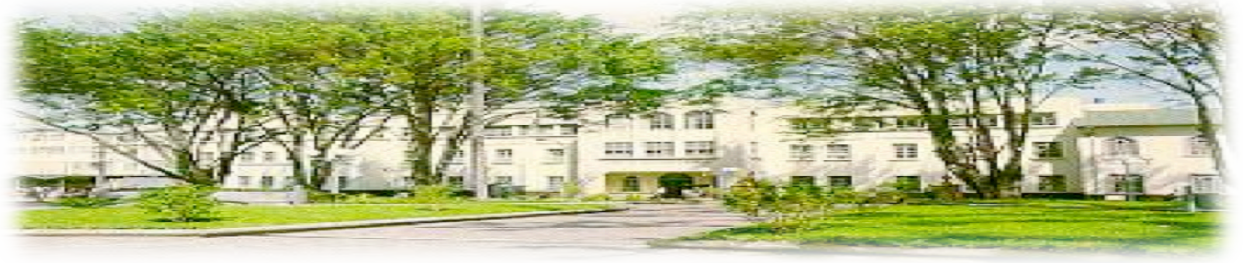

Haspital San Zuan de Dias, San Jasé. Costa Rica. Fundada en 1845

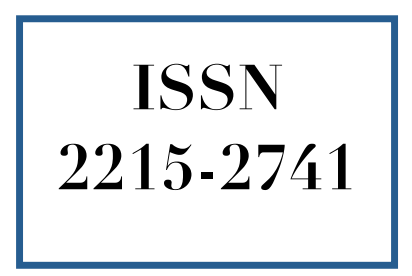

$\begin{array}{ll}\text { Recibido: } & 16 / 09 / 2014 \\ \text { Aceptado: } & 30 / 11 / 2014\end{array}$

Andrea Medina Méndez ${ }^{1}$

Kenneth Sanabria Jiménez ${ }^{2}$

David Ávalos Chacón ${ }^{3}$

${ }^{1}$ Médica Residente de Medicina Paliativa Sistema de Estudios de Posgrado. UCR-CENDEISS-CCSS. Correo electrónico: andreamedinacr@hotmail.com

${ }^{2}$ Médico Asistente especialista en Psiquiatra. HCG. CCSS. Profesor del postgrado de Medicina Paliativa Sistema de Estudios de Posgrado. UCR-CENDEISSS

${ }^{3}$ Médico asistente especialista en Geriatría y Gerontología. Médico Paliativista del HNGG. CCSS.Director del postgrado de Medicina Paliativa. SEP UCR-CENDEISSS.

\section{RESUMEN}

Justificación y Objetivos: La demanda de servicios de Cuidados Paliativos en nuestro país ha ido creciendo de forma muy importante. Sin embargo a nivel nacional, no hay estudios descriptivos de la población del Centro Nacional del Control del Dolor y Cuidados Paliativos (CNCDYCP). Método: Estudio observacional, retrospectivo, descriptivo de registros médicos.
Se tomó una muestra. Se utilizó una hoja de recolección de los datos. Resultados: La muestra fue de 484 expedientes donde la edad promedio fue de 69 años, predominaron las mujeres (53\%) y casados (50\%). El 97\% de la población era de San José. La neoplasia gástrica fue la predominante en la población general; la especialidad que más refirió fue Medicina Paliativa $(22 \%)$ y el centro que más refirió fue el Hospital Calderón Guardia (46.9\%). Sólo un 5\% del total de pacien- 
tes recibió atención psiquiátrica, un $44 \%$ de psicología; un $15.08 \%$ de terapia respiratoria; $3.71 \%$ de Terapia Física; $17.76 \%$ de nutrición. Un $80.78 \%$ recibió valoración de visita domiciliar. El $43 \%$ de la población estudiada estuvo entre 2-30 días en el CNCDYCP. Conclusión: El CNCDYCP cumple con la mayoría de los servicios que mencionan las instituciones y guías internaciones que debería de contar una Unidad de Cuidados Paliativos Especializados; sin embargo no cuenta con un Hospital de Día ni Trabajo Social.

\section{PALABRAS CLAVE \\ Paliativo, temporal, cáncer.}

\section{ABSTRACT}

Justification for and Objectives: The demand for Palliative Care in our country has grown very significantly. But no national studies descriptive studies of the population of the National Center for Pain Control and Palliative Care (CNCDYCP). Methods: Observational, retrospective, descriptive study of medical records. A sample was taken. A sheet of data collection was used. Results: The sample consisted of 484 records where the average age was 69 years, women predominated $(53 \%)$ and married $(50 \%) .97 \%$ of the population was in San Jose. Gastric neoplasia was predominant in the general population; The most referred specialist was Palliative Medicine (22\%) and the center with most talked was the Hospital Calderón Guardia (46.9\%). Only 5\% of all patients received psychiatric care, $44 \%$ of psychology; $15.08 \%$ of respiratory therapy; $3.71 \%$ of physical therapy; $17.76 \%$ of nutrition. A score of $80.78 \%$ received home visits. $43 \%$ of the study population was between 2-30 days in the CNCDYCP. Conclusion: CNCDYCP meets most of the services mentioned institutions and admissions guides should tell a Specialist Palliative Care Unit; however does not have a day hospital or social work.

\section{KEY WORDS}

Palliative, terminal cancer.

\section{INTRODUCCIÓN}

A nivel de América Latina (AL) y el Caribe se espera que en el año 2020 ocurran 840.000 defunciones por cáncer ${ }^{(1)}$. Se estima que en los próximos años cerca de 1 millón de personas necesitarán Cuidados Paliativos (CP) en la zona ${ }^{(1)}$.

Sin embargo, al ser la Medicina Paliativa (MP) una especialidad relativamente nueva en el país (alrededor de 20 años) se cuenta con muy pocas investigaciones en esta área y la demanda de los servicios de CP en nuestro país ha ido aumentando ${ }^{(2)}$.

Según el Ministerio de Salud (MS) de Costa Rica (CR) a través de la Dirección de Vigilancia de la Salud con base en datos del Instituto Nacional de Estadística y Censo (INEC); el cáncer aportó para el 2012 la mitad de las muertes prematuras $^{(3)}$. Para el 2012 la incidencia de las diez localizaciones de cáncer más frecuentes, en orden descendente, fueron: piel, próstata, mama, cérvix, estómago, tiroides, colon, sistema hematopoyético, ganglio linfático y pulmón ${ }^{(3)}$.

El objetivo de los $\mathrm{CP}$ es anticipar, prevenir y reducir el sufrimiento y apoyar la mejor calidad de vida posible para los pacientes y sus familias ${ }^{(4,5)}$; independientemente de la etapa de la enfermedad y las terapias dirigidas a prolongar la vida $^{(4)}$. Asimismo, ofrecer apoyo psicológico y social al paciente y a sus cuidadores para posibilitar el alta o traslado a otro entorno dónde continuar con su cuidado ${ }^{(5)}$.

Los CP deben ser iniciados por el equipo primario de oncología y luego aumentada por la colaboración con el avance de un equipo interdisciplinario de expertos en $\mathrm{CP}^{(4)}$.

En CR, en 1991 inició la primera Clínica de Control del Dolor y Cuidados Paliativos ${ }^{(6)}$ que años después cambió su nombre por el de Centro Nacional de Control del Dolor y Cuidados Paliativos $(\mathrm{CNCDYCP})^{(6)}$. El Colegio de Médicos y Cirujanos de CR; reconoció en el 2008 la MP como una especialidad y el CENDEISSS abrió en 2011 el postgrado en MP de adultos ${ }^{(7)}$ del cual está próxima a egresar la segunda generación.

La medicina nació cuando ninguna enfermedad era curable pero todos los enfermos eran cuidables $^{(8)}$. El médico en la antigüedad sólo ejercía el 
cuidar $^{(8)}$. El médico actual es "adicto" a curar. No se debe olvidar que la medicina sin caridad es inhumana y sin ciencia peligrosa. Como siempre en el punto medio está la virtud.

Hay diversos niveles de organización en CP definidos en relación con su complejidad ${ }^{(5,9,10)}$.

1. Básicos o primarios: Son los cuidados que se entrega por los médicos generales ${ }^{(9)}$.

2. Especializados (CPE): proporcionados por expertos; un equipo multiprofesional capacitado. Los CP deben constituir el principal objetivo de su trabajo diario $^{(5)}$.

\section{MATERIALES Y MÉTODOS}

Es un estudio observacional, retrospectivo, descriptivo de registros médicos. Realizado en el CNCDYCP durante el 2013. Se trabajó con una muestra de 484 expedientes. La estadística descriptiva se realizó usando el programa SPSS y Stata.

Luego de la autorización de parte del CLOBI del HCG; se solicitó al archivo clínico del hospital los expedientes y se llenó una hoja de recolección de datos por expediente que cumpliera los criterios de inclusión.

Los criterios de exclusión fueron:

- Menores de 18 años.

- Paciente no portador de una enfermedad oncológica terminal.

- Expedientes extraviados; con letra ilegible: mal microfilmados o incompletos.

- Ingresados al CNCDYCP durante 20102012 pero que todavía no han fallecido.

- Fallecidos durante 2010-2012 pero que ingresaron antes de 01 de Enero del 2010 o después del 1 de Enero del 2013.

Se tomó como fecha de ingreso al CNCDYCP a la fecha de la primera cita en Consulta Externa (CE) con un médico (directa o indirectamente) en el CNCDYCP. La fecha de ingreso al programa de VD fue la fecha en que se realizó la referencia al programa de VD. Mientras que la fecha de defunción fue tomada del Registro Nacional Civil.

El objetivo general del estudio fue describir los servicios brindados a los usuarios mayores de 18 años con enfermedad oncológica terminal en el CNCDYCP, desde su ingreso hasta su fallecimiento, 2010 - 2012. Los objetivos específicos fueron: precisar las principales características socio-demográficas y de condición de salud oncológica; identificar las enfermedades oncológicas e identificar las características de la atención brindada a la población de estudio.

\section{Limitaciones y posibles sesgos del estudio}

Entre las limitaciones para el desarrollo de esta investigación está la falta de información nacional sobre el tema. El acceso a los expedientes. La disponibilidad del tiempo para la recolección de datos. La falta de algunos datos en los expedientes así como la pobre información estadística que el CNCDYCP tiene.

\section{RESULTADOS}

La edad promedio fue de 69 años, con una desviación estándar de alrededor de 15 años. Se observó un paciente con una edad de 18 años, siendo la más baja y otro paciente con 100 años de edad (Tabla 1).

Un 53\% correspondió a mujeres (Tabla 1). Un $50 \%$ estaban casados o casadas; seguido de la viudez con un $21 \%$ y los solteros o solteras con un $16 \%$ (Tabla 1$)$.

Se encontró que el $97 \%$ correspondió a pacientes residentes de San José (Tabla 1), de los cuales el $27 \%$ venían del cantón Central de San José, 16\% de Goicoechea y un 9\% de Curridabat. Los distritos que pertenecen al área de atracción del HCG representaron el $67.76 \%$. Un $20.86 \%$ pertenecían al área de atracción del HSJD y un 9.09\% al Hospital México (HM).

La especialidad que más refirió al CNCDYCP fue Medicina Paliativa (22\%), en segundo lugar la Oncología Médica con $18 \%$; seguida de la Oncología Quirúrgica con un 8\% (Gráfico 1). 
Tabla N 1. Estadísticas descriptivas para variables socioeconómicas y el valor del Karnofsky promedio de ingreso y de VD

\begin{tabular}{lccccc} 
Variable & $\begin{array}{c}\text { Edad } \\
\text { promedio }\end{array}$ & Cantidad & Porcentaje & $\begin{array}{c}\text { Karnofsky de } \\
\text { ingreso }\end{array}$ & $\begin{array}{c}\text { Karnofsky } \\
\text { VD }\end{array}$ \\
\hline Mujer & 68,27 & 257 & 53,1 & 67,53 & 55,65 \\
Hombre & 70,71 & 227 & 46,9 & 66,96 & 56,59 \\
Soltero & 66,39 & 79 & 16,3 & 67,85 & 57,41 \\
Unión libre & 62,45 & 20 & 4,1 & 71,11 & 57,50 \\
Divorciado & 61,13 & 39 & 8,1 & 73,43 & 60,37 \\
Viudo & 80,08 & 103 & 21,3 & 61,84 & 53,51 \\
Separado & 60,67 & 3 & 0,6 & 76,67 & 65,00 \\
Casado & 67,87 & 240 & 49,6 & 68,00 & 55,91 \\
San José & 69,39 & 469 & 97,1 & 67,32 & 56,15 \\
Cartago & 72,56 & 9 & 1,9 & 62,50 & 51,43 \\
Heredia & 69,00 & 3 & 0,6 & 80,00 & 60,00 \\
Guanacaste & 51,00 & 1 & 0,2 & 70,00 & 70,00 \\
Limón & 82,00 & 1 & 0,2 & 50,00 & 50,00 \\
Casa & 69,52 & 465 & 96,1 & 67,04 & 56,03 \\
Hospital & 66,89 & 19 & 3,9 & 72,65 & 57,92 \\
Total & $\mathbf{6 9 , 4 3}$ & $\mathbf{4 8 4}$ & $\mathbf{1 0 0 , 0 0}$ & $\mathbf{6 7 , 2 6}$ & $\mathbf{5 6 , 0 9}$ \\
\hline
\end{tabular}

Fuente: revisión de expedientes.

Gráfico 1. Especialidad que más refiere al CNCDYCP en números absolutos.

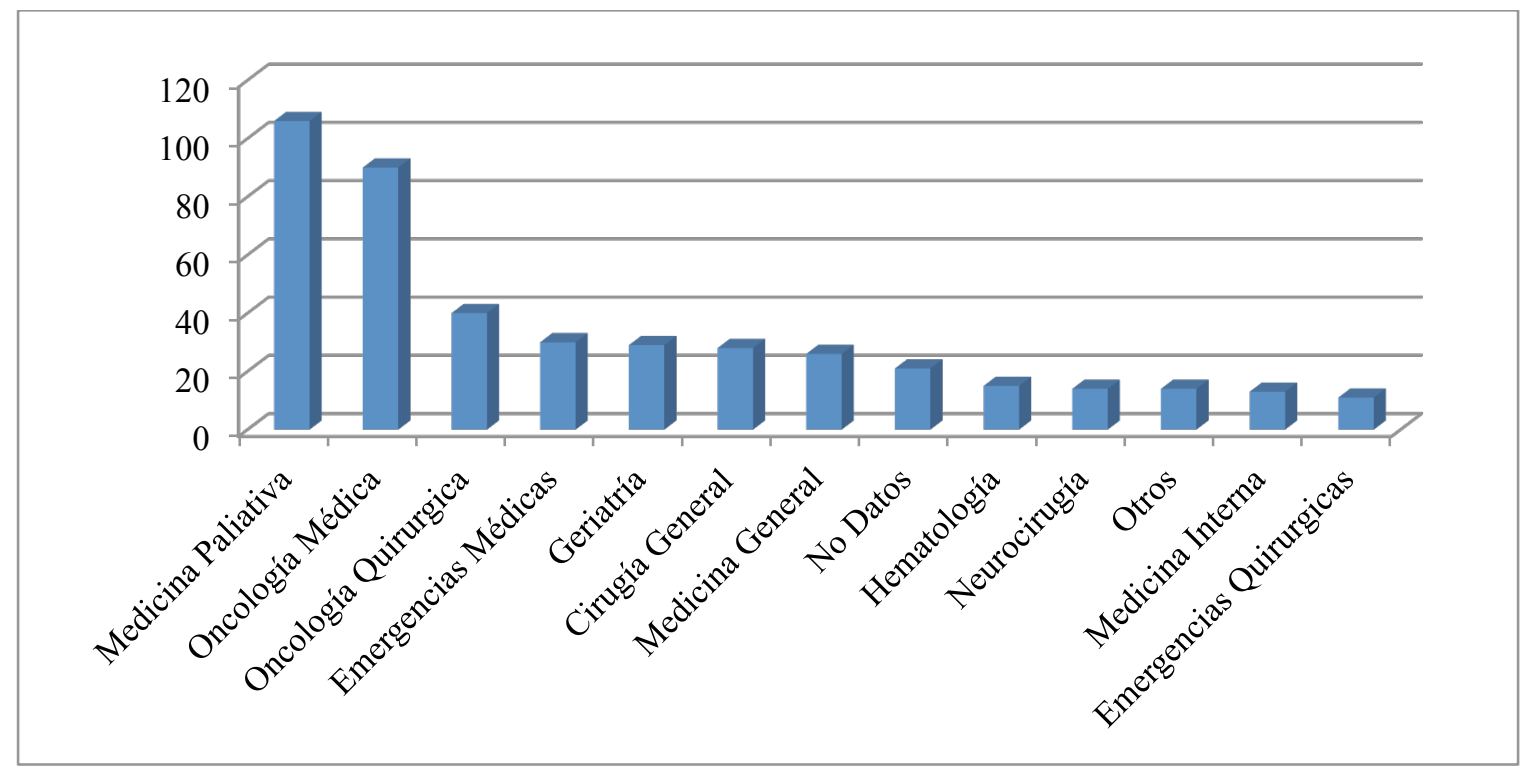

Fuente: revisión de expedientes. 
El principal centro que refirió hacia el CNCDYCP fue el HCG, con un $46.90 \%$; seguido por el HM con un 15.01\%. El CNCDYCP y HSJD ocuparon la tercera posición (Gráfico 2) Las referencias hechas del CNCDYCP hacia el
CNCDYCP, son los pacientes que el personal médico captó durante las interconsultas médicas realizadas a solicitud de médicos tratantes del paciente.

Gráfico 2. Distribución de los Centros Médicos y Especialidades que refieren al CNCDYCP en números absolutos.

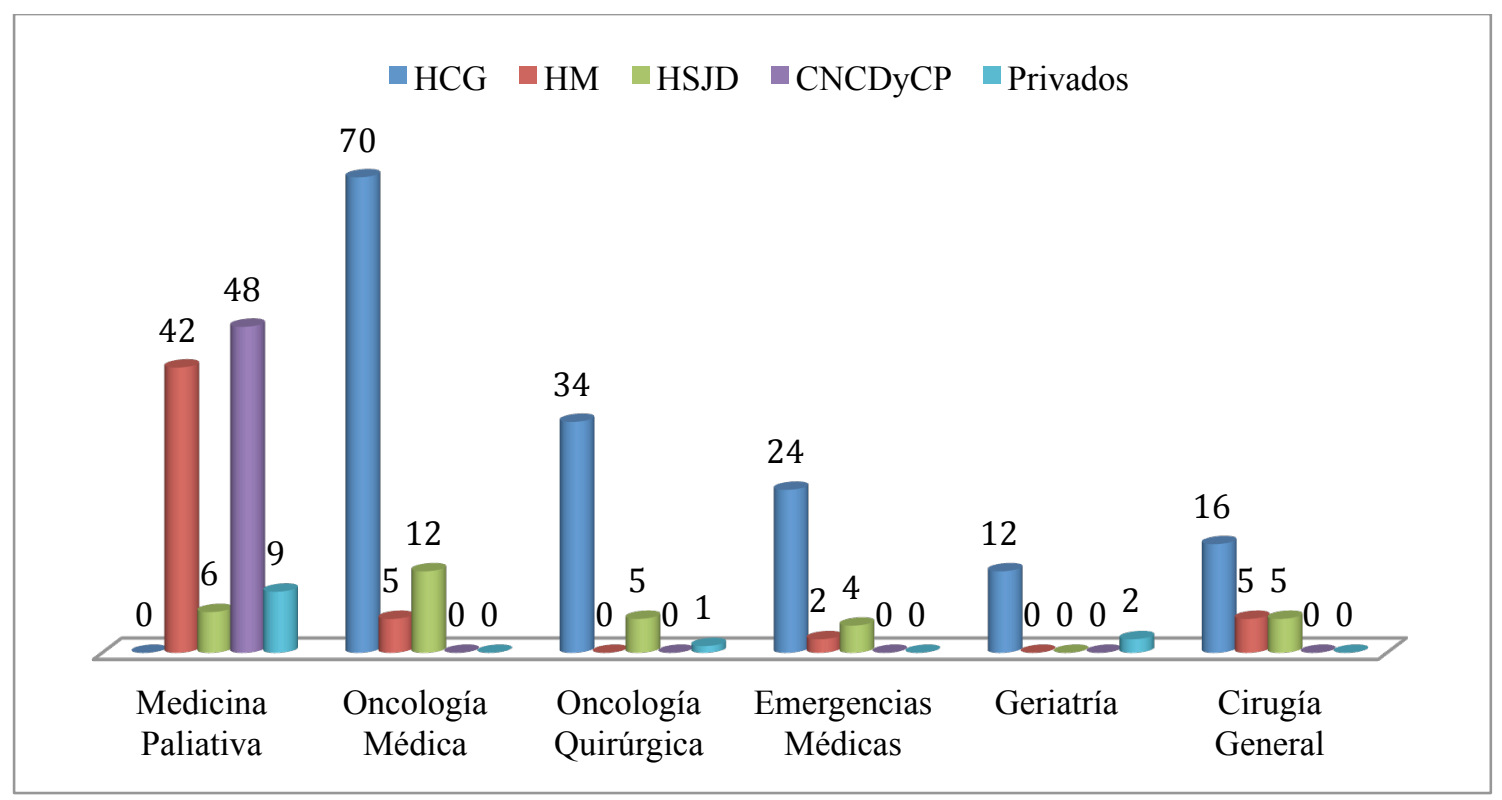

Fuente: revisión de expedientes.

E1 96.07\% de la población falleció en su lugar de habitación y sólo el 3.92\% lo hicieron en algún hospital central de San José, no se identificó cuál (Tabla 1).

En el Karnofsky de ingreso al CNCDYCP predominó la puntuación entre 40 y 69 puntos, con un 52\%. La misma distribución se observó en el Karnofsky de ingreso al programa de VD donde la mayoría de la población estaba entre los $40 \mathrm{y}$ 69 puntos $(80 \%)$.

La neoplasia gástrica fue la principal encontrada en la población estudiada, con 70 casos $(14.46 \%)$. La segunda neoplasia más frecuente fue la neoplasia del intestino grueso y la de híga- do y vías biliares $(9.41 \%)$, seguidas por las neoplasias de mama $(7.02 \%)$.

Las consultas de emergencias/filtros (consulta médica no programada) las necesitaron 105 pacientes. El servicio que más se brinda atención es el de CE con un $88.84 \%$; en segundo lugar fue el de VD con un $80.78 \%$ y en tercer lugar el servicio de psicología con un 43.59\% (Gráfico 3). Por el contrario, el servicio menos utilizados por la población estudiada fue la Terapia Física (TF) con apenas el 3.71\% (Gráfico 3).

Sólo 54 pacientes no fueron atendidos en CE y entraron directamente al programa de VD. 
Gráfico 3. Porcentaje de pacientes atendidos en cada servicio.

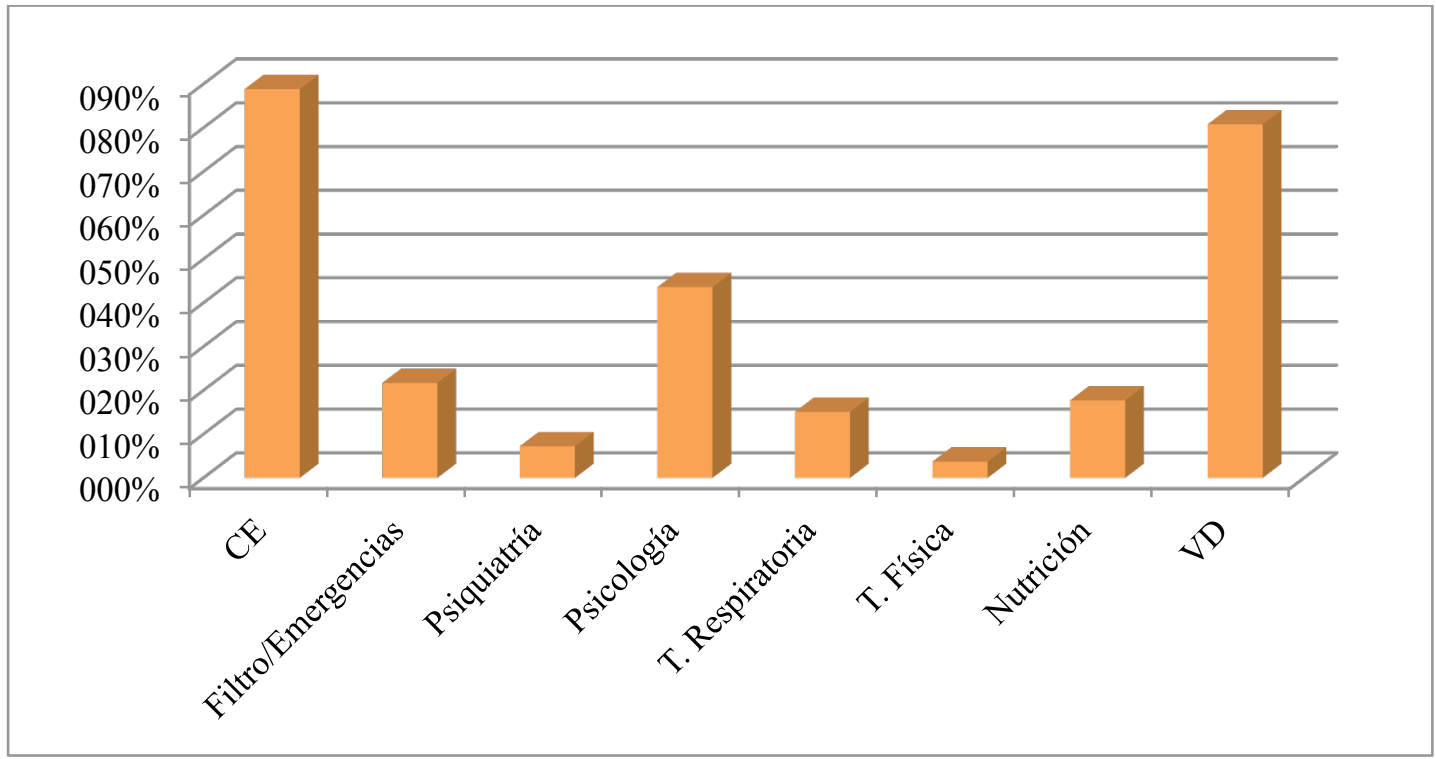

Fuente: revisión de expedientes.

El $43 \%$ de la población estudiada estuvo entre 230 días en el CNCDYCP; el 40\% estuvo entre 31-182 días y el 10\% entre 183-364 días (Gráfico 4). A nivel de la CE médica, la estancia promedio desde la fecha de ingreso hasta la fecha en que fue referido a VD; fue de: $54 \%$ de la población sólo estuvo un día en dicho servicio; el $20 \%$ estuvo entre 31-182 días y el 19\% entre 2-30 días. A nivel del programa de VD, desde su ingreso a dicho programa y hasta el fallecimiento del paciente; el 59\% de los pacientes estuvo entre 2-30 días; el 33\% entre 31-182 días y el 4\% entre 183-364 días.

Gráfico 4. Porcentaje de estancia promedio desde el ingreso al CNCDYCP hasta el fallecimiento

- 1 día $\quad 2$ - 30 días $\square$ 31-182 días $\quad 183$-364 días $\quad 365$ - 729 días $\quad 730$ - 1094 días

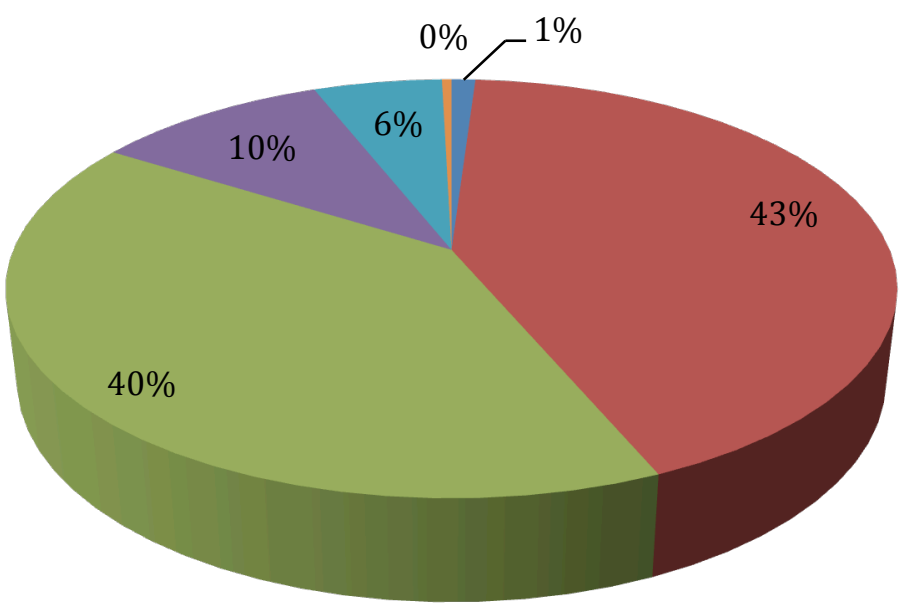

Fuente: revisión de expedientes. 


\section{DISCUSIÓN}

La edad media fue de 69.41 años (Tabla 1); lo cual sugiere que se trata de una población envejecida que puede tener múltiples comorbilidades asociadas a su enfermedad neoplásica y ésto podría implicar un aumento en las consultas a diferentes centros de salud; por la descompensaciones de dichas comorbilidades así como un consumo mayor de fármacos.

Queda para futuros estudios, el establecer por qué el género femenino es el predominante.

Hay un alto porcentaje de pacientes casados y esto se puede traducir en beneficios para los pacientes ya que contarían con un apoyo sentimental y emocional directo.

Al ser el CNCDYCP un entre nacional, no tiene población adscrita $\mathrm{y}$ debe asumir, siempre $\mathrm{y}$ cuando su ámbito administrativo y organizativo se lo permita, otras poblaciones. Como era de esperar; la provincia de San José es la principal proveedora de los pacientes terminales del CNCDYCP.

El HM no cuenta con programa de VD, por lo que cuando la población oncológica terminal del área de atracción propia de dicho centro amerita VD, debe ser referido al CNCDYCP para su manejo. Dicha población, representa apenas el $9.09 \%$ de total estudiado. Por el contrario, a pesar de que el HSJD sí cuenta con un programa de VD se detectó que un $20.86 \%$ de la población estudiada pertenecían al área de atracción del HSJD. Lo cual deja la interrogantes del por qué para futuras investigaciones.

Como era de esperar, el principal lugar de dónde viene referidos la mayoría de la población del CNCDYCP es el HCG (46.90\%) y las principales especialidades que refiere son: Medicina Paliativa (21.90\%), Oncología Médica (18.54\%) y Oncología Quirúrgica (8\%) (Gráfico 2).

El $43 \%$ de los pacientes tuvo control en el CNCDYCP (incluyendo CE médica y VD) por más de 1 día pero menos de 30 días, lo cual nos habla de lo tarde que se están realizando las intervenciones de los CP en la población del CNCDYCP estudiada y lo rápido que hay que actuar cuando los pacientes ingresan (Gráfica 4).
E1 54\% de los pacientes fue referido al programa de VD el mismo día en que ingresaron al CNCDYCP, lo cual sugiere que desde su ingreso eran pacientes con una expectativa de vida limitada, esto es apoyado por el Karnofsky de ingreso, que fue menor de 70 puntos en el $53 \%$ de la población (233 pacientes).

De los pacientes que fallecieron en su lugar de habitación la edad promedio era de 69.52 años; mientras que los que fallecieron en el hospital eran un poco más jóvenes con 66.89 años.

Las tres principales neoplasias detectadas en la población del estudio fueron: gástrica, del intestino grueso e hígado y vía biliar en el segundo lugar y la de origen desconocido en el tercero. Esta distribución difiere a la mencionada por el $\mathrm{MS}^{(3)}$ donde se encuentra en primer lugar la próstata seguida de gástrica y mama.

De la población que recibió atención del servicio de psiquiatría (35 pacientes); en promedio recibieron 1.2 consultas. Mientras que de los pacientes que fueron atendidos por psicología; en promedio tuvieron 1.8 consultas.

No queda claro por qué a más del $50 \%$ de la población analizada no se le brindó atención psicológica $(53.1 \%)$ a pesar de que existe un programa de visita psicológica domiciliar. Hubo un $2 \%$ de los pacientes fallecieron antes de que se les lograra realizar la primera VD.

Sólo un 15.1\% recibió atención por el servicio de TR y en promedio esos pacientes tuvieron 1.4 consultas. Es llamativo que solo el $43.2 \%$ de los pacientes con neoplasias de pulmón y mediastino recibieron atención por parte del servicio de TR del CNCDYCP, sobre todo porque son pacientes que van a presentar disnea ${ }^{(11)}$ al final de su enfermedad y la terapia respiratoria forma parte del manejo no farmacológico de dicha disnea ${ }^{(12)}$.

Un $3.71 \%$ de los pacientes recibió atención por parte del servicio de TF y en promedio cada paciente recibió 1.8 consultas. A pesar de que en el estudio de Abadía ${ }^{(13)}$ que se desarrolló en pacientes costarricenses del CNCDYCN y población española y evidenció los beneficios del ejercicio físico en el paciente oncológico, como mejorar la fatiga y el mantenimiento de la escala de Barthel durante un período de tiempo mayor. 
El CNCDYCP es el ente institucional público más especializado (tercer nivel de atención) y no cuenta o al menos no contaba al momento de la realización del estudio, con un servicio de Hospital de Día ni de Trabajo Social. Dos tipos de consulta habituales de un UCPE según la SECPAL y la OMS ${ }^{(10,14)}$. Tampoco cuenta con una Unidad de Enfermería a domicilio, coordinadores de apoyo espiritual, capellanes, especialistas en linfedema y/o terapia ocupacional ${ }^{(5)}$.

\section{CONCLUSIONES Y RECOMENDACIONES}

Se encontró que en la población analizada el promedio de la edad fue de 69.41 años; predominaron las mujeres (53\%). La gran mayoría estaba casado o casada $(50 \%)$.

El 98.14\% de la población vivía en la Provincia de San José, de los cuales el cantón Central $(27.36 \%)$ fue el principal y de éste los distritos que pertenecen al área de atracción del $\mathrm{HCG}$ representaron el $67.76 \%$ (328 pacientes). El $96.07 \%$ de los casos fallecieron en el lugar de habitación.

El HCG refirió el 46.90\% (227 pacientes) y es la MP la especialidad que más refiere $(21.90 \%)$ al CNCDYCP.

El Karnofsky de ingreso al CNCDYCP en el $52 \%$ de los casos se encontraba entre 40-69 puntos (227). Mientras que el Karnofsky de ingreso al programa de VD en el $80 \%$ de los casos fue entre 40-69 puntos.

La estancia promedio desde la primera cita en CE médica del CNCDYCP hasta el fallecimiento en el 43\% de los casos fue entre 2-30 días.

La estancia promedio de los pacientes que estuvieron siendo atendidos en la CE médica y hasta la referencia al programa de VD en el $54 \%$ de la población estudiada fue de solo 1 día. Mientras que la estancia promedio en el programa de VD desde que fue referido al fallecimiento del paciente fue en el 59\% de los casos entre 2-30 días.

En los hombres la neoplasia más frecuente fue la gástrica $(9,5 \%)$ mientras que en las mujeres fue la neoplasia de mama $(7.02 \%)$.

En el CNCDYCP, no hay hospital de día ni TS.

\section{REFERENCIAS BIBLIOGRÁFICAS}

1. Bruera E De Lima L. Cuidados Paliativos. Guías para el manejo clínico. [Internet]. Organización Panamericana de la Salud; Recuperado a partir de: http://www.paho.org/HQ/index.php?option= com_docman\&task $=$ doc view\&gid $=18717$ \&Itemid $=$

2. Salas I. Programa académico de la especialidad en Medicina Paliativa en adultos [Internet]. CENDEISSS; 2010. Recuperado a partir de: http://www.cendeisss.sa.cr/posgrados/docum entos/Programas/Medi \%20Pali_Adultos.pd

3. Ministerio de Salud, Costa Rica. Memoria Institucional 2012 [Internet]. El Ministerio; 2013. Recuperado a partir de: http://www.ministeriodesalud.go.cr/sobre m iniste-

rio/memorias/memoria institucional 2012/ UMI memoria institucional 2013.pdf

4. National Comprehensive Cancer Network. NCCN Guidelines Version 2.2013 Palliative Care [Internet]. 2013. Recuperado a partir de:

http://www.nccn.org/professionals/physician gls/pdf/palliative.pdf

5. Radbruch L Payne S Bercovitch M Caraceni A. Libro blanco sobre normas de calidad y estándares de cuidados paliativos de la Sociedad Europea de Cuidados Paliativos [Internet]. 2012. Recuperado a partir de: http://www.secpal.com/b archivos/files/mon ografia secpal 00.pdf

6. López E Morales C. Historia de los Cuidados Paliativos en Costa Rica dentro de la Seguridad Social. Med Paliativa. 2005;12 (2):92-98.

7. Pastrana T De Lima L. Atlas de Cuidados Paliativos en latinoamérica [Internet]. IAHPC Press; 2012. Recuperado a partir de: http://cuidadospaliativos.org/uploads/2012/1 0/atlas/Atlas\%20de\%20Cuidados\%20Paliati vos\%20de\%20Latinoamerica.pdf

8. Sanz Y. Historia de la Medicina Paliativa. Med Pal (Madrid). 1999;6(2):82-88.

9. Cinnamon J Schuurman N Crooks VA. $A$ method to determine spatial access to specialize palliative cares services using GIS. BMC Health Serv Res. 2008;8(1):140.

10. DOC_WHOCC ICO Definición Cuidados Paliativos ESP - DOC_WHOCC ICO Definición Cuidados Paliativos ESP.pdf [Internet]. [citado 6 de noviembre de 2013]. Re- 
cuperado a partir de: http://www20.gencat.cat/docs/salut/Minisite/ ICO/Professionals/Documents/QUALY/Arx ius/DOC WHOCC $\% 20 \mathrm{ICO} \% 20$ Definici $\% \mathrm{C}$ 3\%B3n $\% 20$ Cuidados $\% 20$ Paliativos $\% 20 \mathrm{ESP}$ .pdf

11. Twycross RG Wilcock A Stark C. Symptom management in advanced cancer. Nottingham: Palliativedrugs.com 2009.

12. Evidence-based practice of palliative medicine. Philadelphia: Elsevier/Saunders; 2013.

13. Abadia K. Ejercicio Físico Como Parte de la Atención de los Pacientes Oncológicos Paliativos [Internet]. 2010. Recuperado a partir

http://gredos.usal.es/jspui/bitstream/10366/8 3192/1/DME Abad\%C3\%ADaCubillo Karl a Ejercicio.pdf

14. Sociedad Española de Cuidados Paliativos. Guía de Cuidados Paliativos [Internet]. SECPAL. Recuperado a partir de: http://www.secpal.com/guiacp/guiacp.pdf

\section{FUENTES DE APOYO}

No hay fuentes de apoyo que declarar.

\section{CONFLICTO DE INTERÉS}

No hay conflicto de interés que declarar. 